\title{
Bag-of-Particles Model for Simulating Tissue, Organs, and Flow
}

\author{
David J. Stahl, Jr. and Norberto Ezquerra \\ Georgia Institue of Technology, College of Computing, Atlanta GA, 30332-0280 \\ $\{$ stahl, norberto\}@cc.gatech.edu
}

\begin{abstract}
We present a physically-based, elastically deformable particle system model that combines the oriented particles used in surfaceonly models, and the unoriented particles used in volume-only simulations into a bag of particles. Multiple species of particles and predefined interspecies parameters determine elastic material properties. Model dynamic behavior and global shape are determined by the response of its particle ensemble to (i) volume forces and surface forces and torques, and (ii) orienting and shaping forces and torques derived from a distance map.
\end{abstract}

\section{Particle Dynamics}

Our system employs four potential functions of particle position and orientation that govern particle behavior: one potential that gathers particles into a shapeless, space-filling conglomeration, two potentials that arrange particles into locally planar surface configurations, and one potential that arranges these planar sections into a global object shape. Since the interior of an object does not intrinsically define its shape but is simply a volume filled with material, for our object interiors we use a form of the potential function frequently used in molecular dynamics simulations [1], the Lennard-Jones (LJ) potential, which models condensed matter as an aggregate collection of particles. This potential reasonably models gases and viscous fluids. Particles subject to only a LJ potential will form close-packed, space-filling aggregates in their minimum energy state, but not surfaces. To form surfaces, additional potentials are needed. For this purpose we use the approach of [2, which defines co-normal $(\mathrm{CN})$ and coplanar (CP) potentials that act to arrange oriented particles into locally planar surface sections. To form non-planar surfaces, we add forces to arrange locally planar regions into the desired global object shape, and torques to orient surface particles to the object's surface normal. These forces and torques are obtained from a distance map [3], which defines the distance from a point to the closest point on a surface. The distance map is computed from a voxel bitmap representation of an object, which, in turn, can be obtained from a variety of representations such as polygonal models, implicit functions, or acquired image data. 


\section{Bags of Particles}

The simplest model requires two different particle types: space-filling unoriented volume particles that aggregate due to LJ forces, and oriented surface particles forming an enclosing surface, which are subject to LJ, CN, CP, and distance map forces and torques. The distance map force acts to keep surface particles in surface voxels, and the distance map torque maintains surface particles oriented to the surface normal. To model multiple elastic materials as bags within bags, particles are assigned a species attribute, and a set of inter-species coefficients are used to weight interparticle forces and torques, establishing different elastic behaviors as a function of particle species. Volume particles are free to move within an enclosing structure, such as blood flow within a vessel.
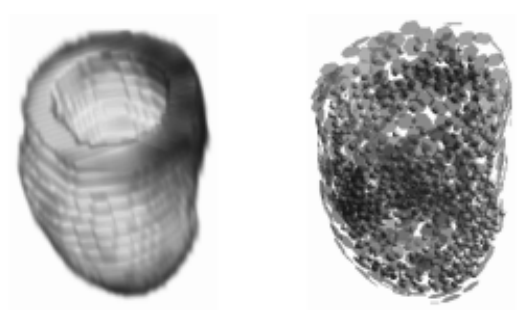

Fig. 1. Left ventricle acquired by PET: volume visualization and bag of particles model.

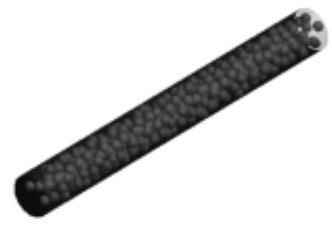

Fig. 2. Bag of particles model of blood flow in a vessel.

\section{References}

1. Rapaport, D.C.: The Art of Molecular Dynamics Simulation. Cambridge University Press, Cambridge, United Kingdom, 1995.

2. Szeliski, Richard and Tonnessen, David.: Surface Modeling with Oriented Particle Systems. Computer Graphics 26 (1992) 185-194

3. Danielsson, Per-Erik: Euclidean Distance Mapping. Computer Graphics and Image Processing. 14 (1980) 227-248 